\title{
Diseño y construcción de un banco de pruebas para ensayos de resistencia estructural de alas y alerones. (1) (1)(9)
}

\section{Design and construction of a test bench for structural resistance tests of wings and ailerons.}

Ing. Guido Torres Mg. ${ }^{1}$, Ing. Mauricio Cruz Mg. ${ }^{2}$, Ing. Stalin Mena Mg. ${ }^{3}$, Egr. Guido Torres Llerena. ${ }^{4}$,Ing. Terán Héctor Mg. ${ }^{5}$ \& Ing. Danilo Zambrano Mg. ${ }^{6}$

\begin{abstract}
.
DOI: https://doi.org/10.33262/cienciadigital.v2i1.7

The project aims to design and build a test bed for testing the structural strength of aircraft wings and spoilers that allows experimentally verify the static loads on the structure of a wing exerted on six points. The wiffletree system (distribution of forces in tree form) by a hydraulic unit generates a tensile force whose pressure is a variable load, to simulate the static loads on the wing in flight (takeoff, horizontal flight and landing ) and thus check the strength of the composition of the different materials used in the aircraft industry for the construction of aircraft wings. The electrohydraulic motor drives the hydraulic cylinder, which transmits the force to the static stress distribution in the plane's wing similar to the wing experiences in horizontal flight test for structural strength. Load cells measure the force on the wing in six specific points and have a communication to a data acquisition board (Arduino ) this information is displayed on a computer that provides status instantly force on the material. So the test began a period of experimentation in the area of materials and structures performing
\end{abstract}

\footnotetext{
${ }^{1}$ Universidad de las Fuerzas Armadas ESPE, Departamento de Ciencias de la Energía y Mecánica,Latacunga, Ecuador,grtorres@espe.edu.ec.

${ }^{2}$ Universidad de las Fuerzas Armadas ESPE, Departamento de Ciencias de la Energía y Mecánica, Latacunga, Ecuador,gmcruz@espe.edu.ec.

${ }^{3}$ Universidad de las Fuerzas Armadas ESPE, Departamento de Ciencias de la Energía y Mecánica, Latacunga, Ecuador, jsmena@espe.edu.ec.

${ }^{4}$ Universidad de las Fuerzas Armadas ESPE, Departamento de Ciencias de la Energía y Mecánica, Latacunga, Ecuador, grtorres3@espe.edu.ec.

${ }^{5}$ Universidad de las Fuerzas Armadas ESPE, Departamento de Ciencias de la Energía y Mecánica, Latacunga, Ecuador, hcteran@espe.edu.ec.

${ }^{6}$ Universidad de las Fuerzas Armadas ESPE, Departamento de Ciencias de la Energía y Mecánica, Latacunga, Ecuador, vdzambrano@espe.edu.ec.
} 
tensile and flexural tests for light industry aeronautics and aerodynamics of the car.

Keywords: Electromagnetic Induction, Heating, Efficiency.

\section{Resumen.}

El proyecto tiene como objetivo diseñar y construir un banco de pruebas para ensayos de resistencia estructural de alas de aviones y alerones que permita comprobar experimentalmente las cargas estáticas sobre la estructura de un ala que se ejercen en seis puntos. El sistema wiffletree (distribución de fuerzas en forma de árbol) mediante un grupo hidráulico genera una fuerza a tracción cuya presión es una carga variable, para simular las cargas estáticas sobre el ala en vuelo (despegue, vuelo horizontal y aterrizaje) y con ello comprobar la resistencia de la composición de los distintos materiales que se utiliza en la industria aeronáutica para la construcción de las alas de los aviones. El motor electro hidráulico acciona al cilindro hidráulico, que transmite la fuerza a la distribución de tensión estática en el ala del avión de forma similar a la que el ala experimenta en vuelo horizontal para la prueba de resistencia estructural. Las celdas de carga miden la fuerza sobre el ala en seis puntos específicos y tienen una comunicación a una tarjeta de adquisición de datos (Arduino) esta información se muestra en un computador que proporciona el estado de fuerza al instante sobre el material. Por lo que el banco de pruebas inicia una etapa de experimentación en el área de materiales y estructuras realizando ensayos a tracción y flexión para el sector de la aeronáutica ligera y la aerodinámica del automóvil.

Palabras Claves: Ingeniería Automotriz, Aeronaves, Alas y Alerones, Banco de Pruebas, Diseño y Construcción

\section{Introducción.}

El proyecto responde a la necesidad de realizar una investigación en el diseño y construcción de alas de aviones y alerones con la finalidad de determinar la resistencia estructural de distintas composiciones de materiales y distintas fórmulas estructurales en la construcción de los mismos.

Este proyecto permite desarrollar investigación y desarrollo autónomos para adquirir y generar conocimientos en el área de la ciencia de materiales y sus aplicaciones. Optimizar las combinaciones de materiales y la fórmula estructural para el diseño de alas de un UAV, es la razón por la cual planteamos el diseño y construcción de un banco de pruebas para ensayos de resistencia estructural de alas y alerones. Esto nos permitirá determinar la mejor combinación y forma del ala, a objeto de obtener la mejor relación de resistencia estructural sobre el peso total del ala, por lo que es necesario, diseñar y construir un banco de pruebas 
para ensayos de resistencia estructural de las alas de aviones y alerones, y verificar su resistencia a los tipos de carga y esfuerzos estáticos a los que se encuentran expuestas.

\section{Desarrollo del proyecto.}

\section{A. Parámetros de diseño.}

El banco de pruebas es capaz de soportar las cargas de prueba sobre el modelo. La carga estática es aplicada por medio de un cilindro hidráulico que soporta sobre la parte superior de la estructura del bastidor, la carga es aplicada sobre la viga superior del sistema de distribución de fuerzas que transmite la carga para simular la aplicación de carga distribuida sobre el sujeto de prueba.

Para la probeta de prueba se considera que uno de los extremos está empotrado y el otro libre. El bastidor está formado por dos columnas apoyadas sobre el piso y sobre estas una viga que es la que soporta al elemento transmisor de carga.

La deflexión permisibe no deberá ser mayor que L/240.

Las cargas que son parte del análisis son:

- Carga muerta: masa propia de la estructura.

- Carga Viva: carga de prueba (se considerá carga máxima)

- La combinación de carga se aplica de acuerdo a lo indicado en la norma ASCE 7.

- Para el analisis se realizará el estudio de una carga distribuida triangular como se observa en la figura 1.

Figura 1: Carga distribuida triangular.

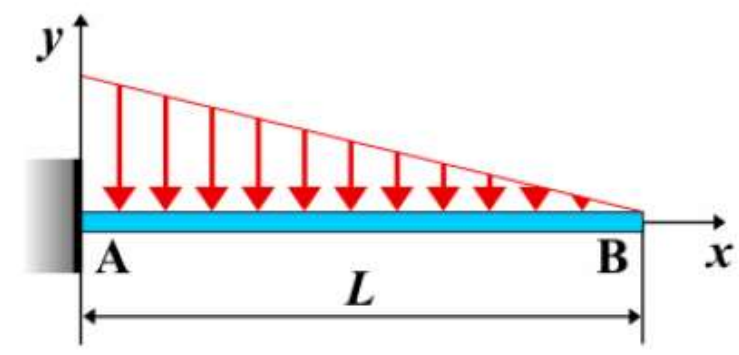

Fuente: Grupo de Investigación.

Fuerza:

$$
W_{x}=\frac{W(L-x)}{L}
$$

\section{Reacciones:}




$$
R_{A}=\frac{W \cdot L}{2}
$$

\section{Esfuerzo cortante:}

$$
V_{A B}=\frac{W}{2 L} \cdot(L-x)^{2}
$$

Momentos flectores:

$$
M_{A B}=\frac{W}{6 L} \cdot(L-x)^{3}
$$

\section{Pendiente:}

$$
\theta_{A B}=\frac{W}{24 \cdot E \cdot I \cdot L} \cdot\left(4 \mathrm{~L}^{2}-6 \mathrm{~L}^{2} \cdot \mathrm{x}+4 \mathrm{Lx}^{2}-\mathrm{x}^{3}\right)
$$

\section{Deflexión:}

$$
Y_{A B}=\frac{\text { W. } x^{2}}{120 \cdot E \cdot I \cdot L} \cdot\left(10 L^{3}-10 L^{2} \cdot x+5 L x^{2}-x^{3}\right)
$$

\section{Donde:}

$\mathrm{W}=$ Fuerza aplicada $(\mathrm{N} / \mathrm{m})$.

$\mathrm{W}_{\mathrm{x}}=$ Fuerza en un punto $\mathrm{x}(\mathrm{N})$.

$\mathrm{R} 1=$ Fuerza reactiva $(\mathrm{N})$.

$\mathrm{V}=$ Esfuerzo cortante $(\mathrm{N})$.

$1=$ Longitud de la viga $(\mathrm{m})$.

$\mathrm{I}=$ Momento de Inercia $\left(\mathrm{m}^{4}\right)$.

\section{B. Análisis de los elementos mecánicos del banco de resistencia estructural de alas y} alerones.

Cada uno de los elementos del banco de pruebas están diseñados y simulados aplicando cargas muertas y cargas vivas con la ayuda de un software CAE para seleccionar el material ASTM A 36 que se encuentra en el mercado de nuestro país.

a) Análisis en la distribución de fuerzas. 
Para el análisis en la distribución de fuerzas se aplica una carga (Ps), que es igual a la sustentación máxima de un ala de avión UNASUR I más la carga muerta de la estructura, y se distribuye esta carga sobre el ala para determinar las fuerzas en cada punto, como se muestra en la figura 2.

Figura 2: Cargas aplicadas.

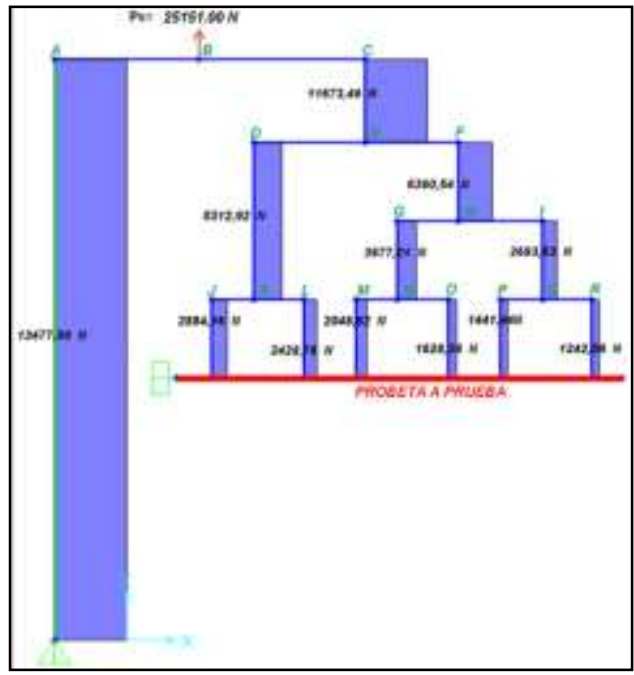

Fuente: Grupo de Investigación.

$$
\mathrm{Ps}=25151 \mathrm{~N}
$$

Figura 3: Tensión de Von Mises.

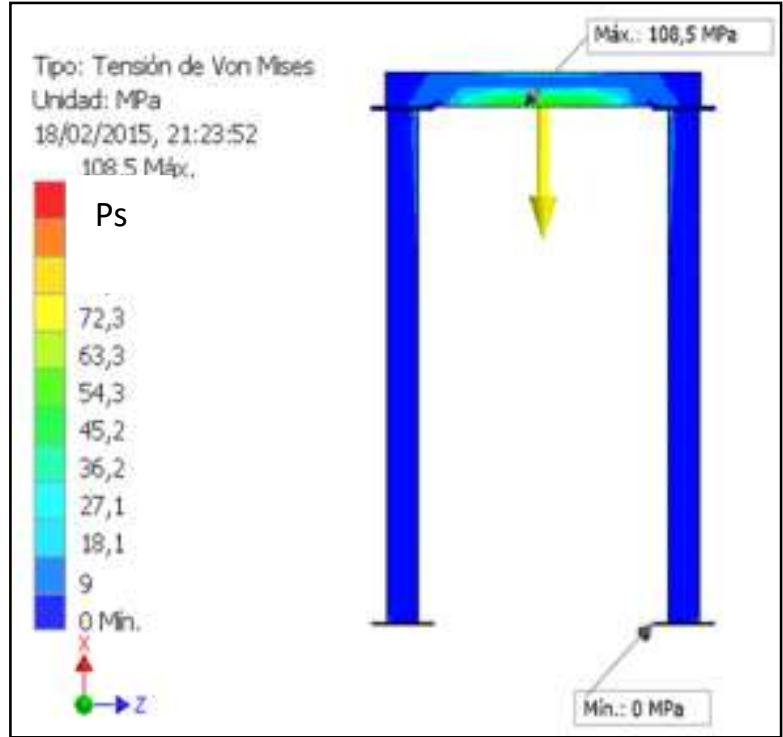

Fuente: Grupo de Investigación. 
En la figura 3 se determina que la estructura del bastidor tiene una tensión de Von Mises máxima de 108,5 MPa. Esta tensión es menor que el límite de elasticidad del material ASTM A36 de $250 \mathrm{MPa}$. Por lo tanto se concluye que puede resistir la tensión máxima a la que está sometido.

b) Sistema hidráulico.

El sistema hidráulico se encarga de suministrar la fuerza a tracción con sus componentes como se indica en la figura 4:

1.- Cilindro hidráulico doble efecto .

2.-Regulador de caudal unidireccional .

3.- Válvula 4/3 tipo tándem - Doble control eléctrico.

4.- Manómetro.

5.- Válvula de seguridad.

6.- Motor eléctrico.

7.- Bomba unidireccional .

8.- Filtro.

9.- Depósito atmosférico.

Figura 4. Circuito Hidráulico.

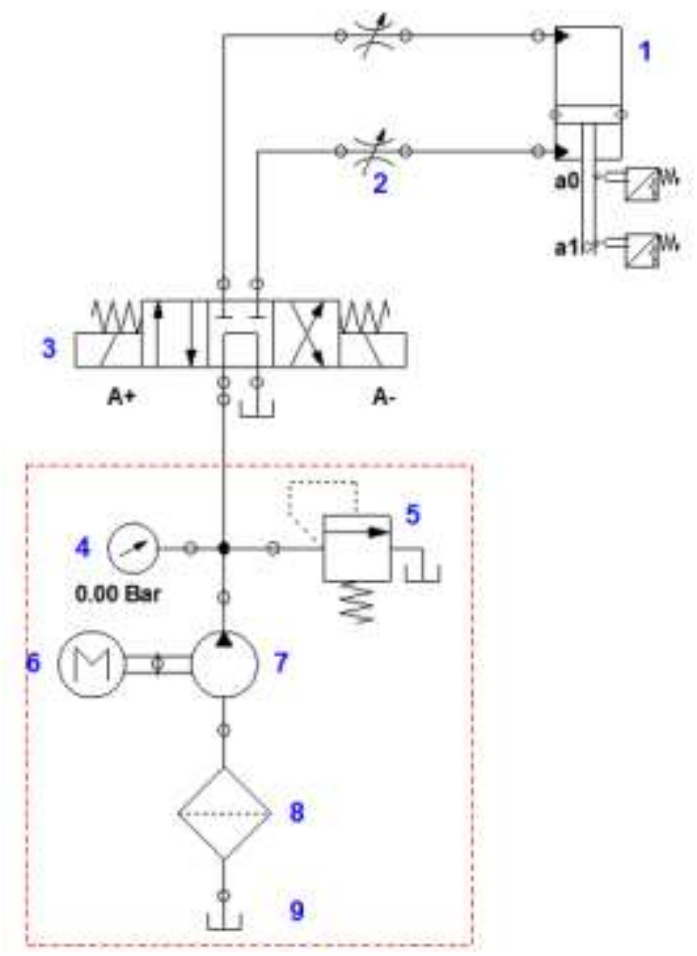

Fuente: Grupo de Investigación 
El área del pistón del cilindro hidráulico es $2,12 * 10^{-3} \mathrm{~m}^{2}$ donde la presión necesaria para generar $25151 \mathrm{~N}$ es $13,79 \mathrm{MPa}$, el caudal de $1,73 \mathrm{gal} / \mathrm{m}$ y la potencia del motor eléctrico sugerida basada en el caudal de la bomba hidráulica y la presión es de 2,02 HP 3HP.

c) Sistema electrónico del banco de pruebas de resistencia estructural de alas.

El sistema de medición de carga consta de una celda de carga, un amplificador de instrumentación, y el conversor A/D dentro de la tarjeta Arduino Leonardo. Los que están conectados para poder obtener los datos de las pruebas mediante un interfaz hacia el computador.

d) Selección de las celdas de carga.

De acuerdo a las cargas obtenidas en cada punto sobre la probeta, que se determina en la distribución de fuerzas de la Figura 2, se seleccionan las celdas de carga de 1000 lb y 500 lb que cumplen con los requerimientos del banco de pruebas.

e) Acondicionamiento de la señal de la celda de carga.

La señal proveniente de la celda de carga no es suficiente para procesarla por lo que es necesario amplificarla con un amplificador de instrumentación AD 620 y un conversor analógico/digital que cuenta el Arduino Leonardo.

\section{Componentes del banco de pruebas.}

Figura 5. Componentes del banco

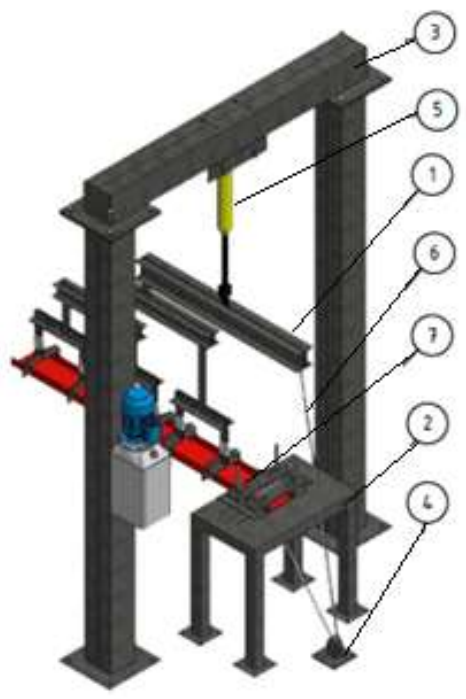

Fuente: Grupo de Investigación.

Tabla 1. Componentes del banco de pruebas. 


\begin{tabular}{ll}
\hline $\begin{array}{l}\mathbf{N}^{\circ} \text { DE } \\
\text { PIEZA }\end{array}$ & ELEMENTO \\
\hline $\mathbf{1}$ & DISTRIBUCIÓN DE FUERZAS \\
$\mathbf{2}$ & MESA DE SUJECIÓN DE LA PROBETA \\
$\mathbf{3}$ & ESTRUCTURA DEL BASTIDOR \\
$\mathbf{4}$ & CONTRAPESO DE ANCLAJE \\
$\mathbf{5}$ & CILINDRO HIDRÁULICO \\
$\mathbf{6}$ & CABLE DE ANCLAJE \\
$\mathbf{7}$ & PROBETA DE PRUEBA \\
\hline
\end{tabular}

Fuente: Grupo de Investigación.

\section{Pruebas de funcionamiento del Banco de Pruebas.}

El banco de pruebas de resistencia estructural de alas fue diseñado para soportar una carga de $25151 \mathrm{~N}$, sus valores ideales de funcionamiento oscilar entre $245 \mathrm{~N}$ a $28694 \mathrm{~N}$ dependiendo del material de la probeta de ensayo.

a) Ensayos de resistencia.

Los ensayos de resistencia estructural son realizados sobre una sección rectangular de madera como se indica en la figura 6.

Tabla 2 Datos de la probeta.

\begin{tabular}{ll}
\hline Datos & \\
\hline Base (b) & $190 \mathrm{~mm}$ \\
Longitud (l) & $1700 \mathrm{~mm}$ \\
Altura (h) & $50 \mathrm{~mm}$ \\
\hline
\end{tabular}

Fuente: Grupo de Investigación.

Figura 6: Probeta de madera. 


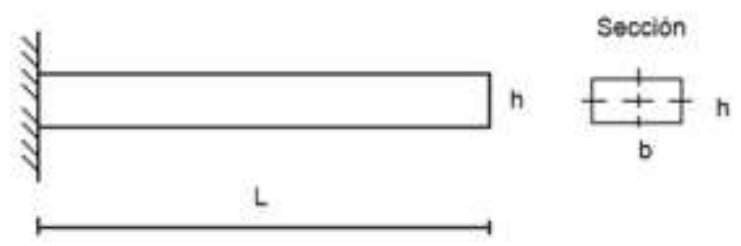

Fuente: Grupo de Investigación.

$$
\mathbf{I}_{\mathbf{x}}=\frac{\mathbf{b} \cdot \mathbf{h}^{\mathbf{3}}}{\mathbf{1 2}}
$$

I: Momento de inercia de la sección transversal de la viga.

Es necesario conocer el momento de inercia de la sección y las propiedades mecánicas de la probeta para el cálculo de la fuerza, reacción, esfuerzo cortante, momento flector y deflexión. El cálculo se desarrolló en función de una carga distribuida triangular como se indica en la figura 1.

Tabla 3 Propiedades mecánicas de la madera.

\begin{tabular}{ll}
\hline Propiedades mecánicas & \\
\hline Densidad & $400 \mathrm{~kg} / \mathrm{m}^{3}$ \\
Módulo de elasticidad & $9000 \mathrm{mpa}$ \\
Resistencia a la tracción & $70 \mathrm{mpa}$ \\
\hline
\end{tabular}

Fuente: (Castaño, En linea).

El análisis se desarrolló en las distancias de ubicación de las celdas de carga que se indican a continuación.

$\mathrm{x}:\left[\begin{array}{llllll}0,14 & 0,49 & 0,69 & 1,04 & 1,24 & 1,59\end{array}\right] \mathrm{m}$

Para las pruebas se tomó como referencia el peso de un avión UAV de $4410 \mathrm{~N}$. La fuerza que se ejerce sobre el ala es de $2205 \mathrm{~N}$ en función del factor de carga $2 \mathrm{G}$ en condiciones de vuelo con turbulencias que pueden afectar a la estructura alar.

b) Prueba I al $1 \mathrm{G}$ de carga.

En esta prueba se consideró una carga de $1 \mathrm{G}$ sobre el ala de $2205 \mathrm{~N}$ y la carga muerta de la distribución de fuerzas de 1445,7 N. Para poder determinar la fuerza que debe ejercer el cilindro hidráulico es necesario sumar la fuerza sobre la probeta más la carga muerta de la distribución de fuerzas obteniendo $3650,7 \mathrm{~N}$. 
En la tabla 4 se puede observar los datos de la carga que debe soportar la probeta a $1 \mathrm{G}$ del peso del avión UAV.

Tabla 4 Fuerza y presión estimada para el ensayo.

\begin{tabular}{cccccc}
\hline & $\begin{array}{c}\text { PRESION } \\
\text { CILINDRO }\end{array}$ & $\begin{array}{c}\text { FUERZA } \\
\text { CILINDRO }\end{array}$ & \multicolumn{2}{c}{$\begin{array}{l}\text { } \text { SENERZA } \\
\text { SENSORES }\end{array}$} & $\begin{array}{c}\text { CARGA } \\
\text { MUERTA }\end{array}$ \\
\hline $\mathbf{n}$ & $(\mathrm{Pa})$ & $(\mathrm{N})$ & $(\mathrm{Lb})$ & $(\mathrm{N})$ & $(\mathrm{N})$ \\
$\mathbf{1} \mathbf{~ G}$ & $1,74 \times 10^{6}$ & 3650,7 & 490 & 2179,63 & 1445,7 \\
\hline
\end{tabular}

Fuente: Grupo de Investigación.

La tabla 5 indica los valores de la carga distribuida obtenidas en las seis celdas de carga en Lb que se muestran en la pantalla de Excel en función del tiempo en la prueba a 3650,7 N.

Tabla 5 Fuerza en las celdas de carga.

\begin{tabular}{|c|c|c|c|c|c|c|}
\hline \multicolumn{7}{|c|}{ TABLA GENERAL DE VALORES OBTENIDOS EN EL TIEMPO } \\
\hline & SENSOR1 & SENSOR2 & SENSOR3 & SENSOR4 & SENSORS & SENSOR6 \\
\hline to1 & 113 & 76 & 42 & 15 & 5 & 2 \\
\hline to2 & 127 & 91 & 55 & 30 & 14 & 5 \\
\hline t03 & 133 & 96 & 58 & 31 & 16 & 7 \\
\hline tou & 152 & 112 & 76 & 43 & 26 & 14 \\
\hline to5 & 163 & 121 & 83 & 47 & 31 & 18 \\
\hline t06 & 167 & 124 & 85 & 52 & 33 & 22 \\
\hline t07 & 165 & 124 & 84 & 56 & 34 & 23 \\
\hline t08 & 168 & 126 & 88 & 56 & 36 & 25 \\
\hline to9 & 167 & 125 & 87 & 54 & 35 & 24 \\
\hline t10 & 166 & 125 & 86 & 54 & 35 & 24 \\
\hline
\end{tabular}

Fuente: Grupo de Investigación.

En la tabla 6 se muestra los datos de las fuerzas obtenidas de las celdas de carga, las fuerzas cortantes, los momentos flexionantes y la deflexión en los seis puntos distribuidos a lo largo de la probeta. Estos resultados se utilizan para determinar las respectivas gráficas y el análisis de la prueba. 
Tabla 6 Análisis de los resultados de la prueba.

\begin{tabular}{cccccc}
\hline PUNTOS & DISTANCIA & $\begin{array}{c}\text { FUERZA } \\
\text { CELDAS DE } \\
\text { CARGA }\end{array}$ & $\begin{array}{c}\text { FUERZAS } \\
\text { CORTANTES }\end{array}$ & MOMENTOS & DEFLEXION \\
\hline P0 & (m) & (N) & (N) & (Nm) & $(\mathbf{m})$ \\
\hline P1 & 0,14 & 738,405 & $-1560,101$ & 811,2523 & 0,001 \\
\hline P2 & 0,49 & 556,028 & $-938,586$ & 378,5631 & 0,005 \\
\hline P3 & 0,69 & 382,547 & $-653,952$ & 220,1640 & 0,009 \\
\hline P4 & 1,04 & 240,204 & $-279,249$ & 61,4347 & 0,018 \\
\hline P5 & 1,24 & 155,688 & $-135,650$ & 20,7996 & 0,023 \\
\hline P6 & 1,59 & 106,757 & $-7,757$ & 0,2844 & 0,034 \\
\hline
\end{tabular}

Fuente: Grupo de Investigación.

\section{b.1) Distribución de fuerzas.}

En la figura 7 se observa la fuerza con respecto a la distancia obteniendo una gráfica que cumple con la distribución de fuerzas que sea mayor en el empotramiento y vaya disminuyendo hacia el extremo libre de la probeta.

Figura 7: Distribución de fuerzas.

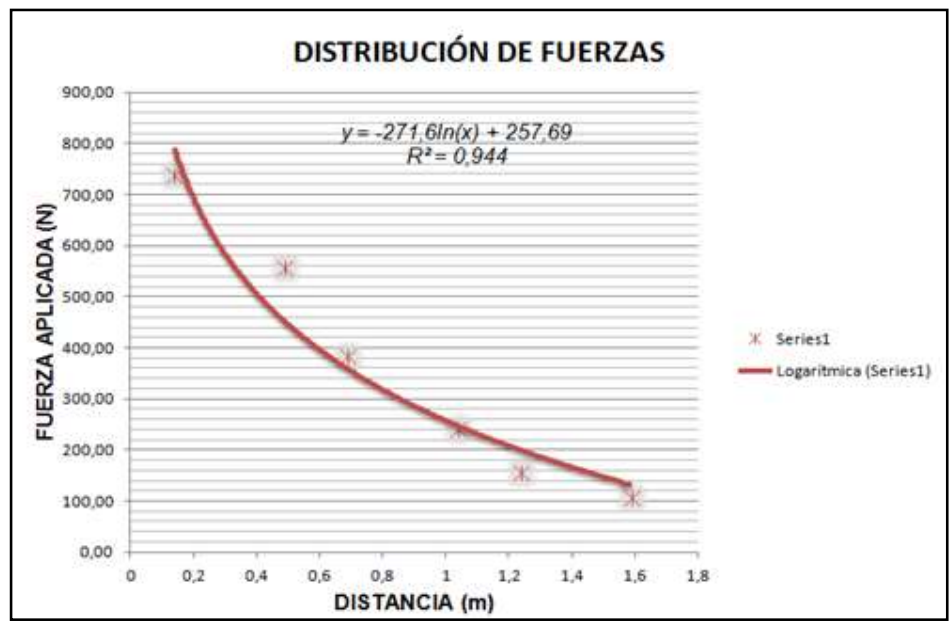

Fuente: Grupo de Investigación. 


\section{b.2) Fuerzas cortantes.}

En la figura 8 determinamos que el punto más crítico es en el empotramiento P0 con un esfuerzo cortante de $-1852,684 \mathrm{~N}$ y el punto $\mathrm{P} 1$ con $-1560,101 \mathrm{~N}$, debido al valor de su carga que se aplica en esa distancia.

Figura 8: Diagrama de esfuerzos cortantes.

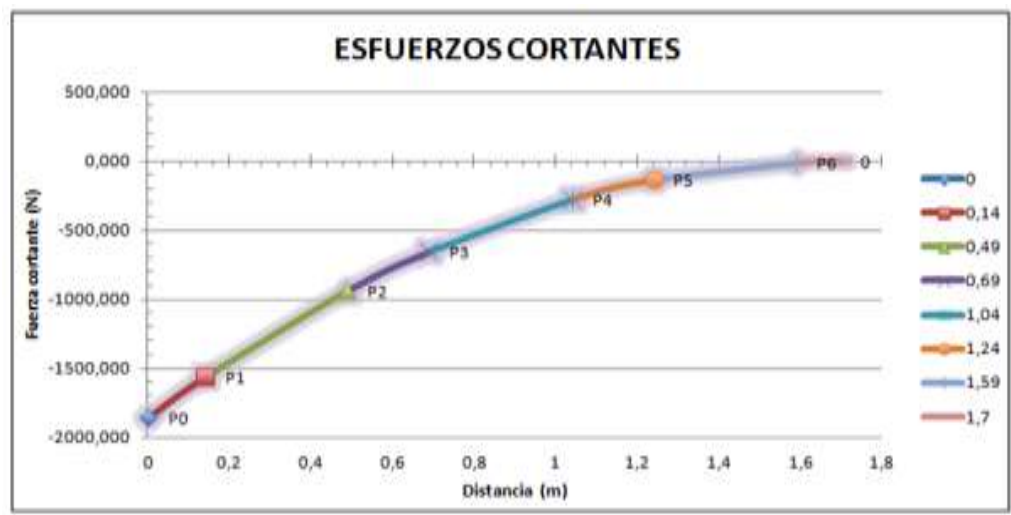

Fuente: Grupo de Investigación.

\section{b.3) Momento flector}

En la figura 9 determinamos que el punto más crítico es en el empotramiento $\mathrm{P} 0$ con un momento flector de $1049,8541 \mathrm{Nm}$ y el punto P1 de 811,2523 , debido a la carga que se aplica en esas distancias.

Figura 9: Momento flector.

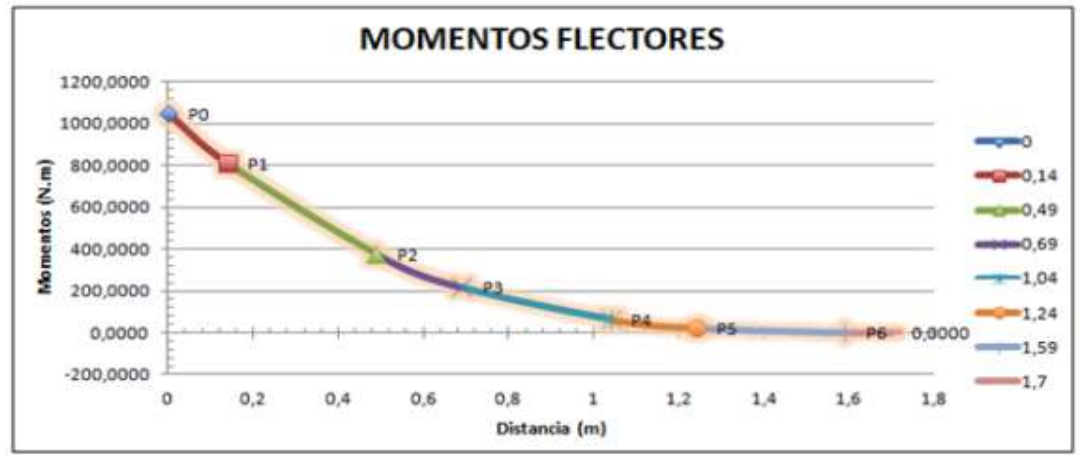

Fuente: Grupo de Investigación. 


\section{b.4) Deflexión.}

En la figura 10 se observa que la deflexión máxima sobre la probeta de madera a una carga de $3650,7 \mathrm{~N}$ se produce en el punto seis obteniendo un valor de $0,034 \mathrm{~m}$.

Figura 10: Curva de la deflexión.

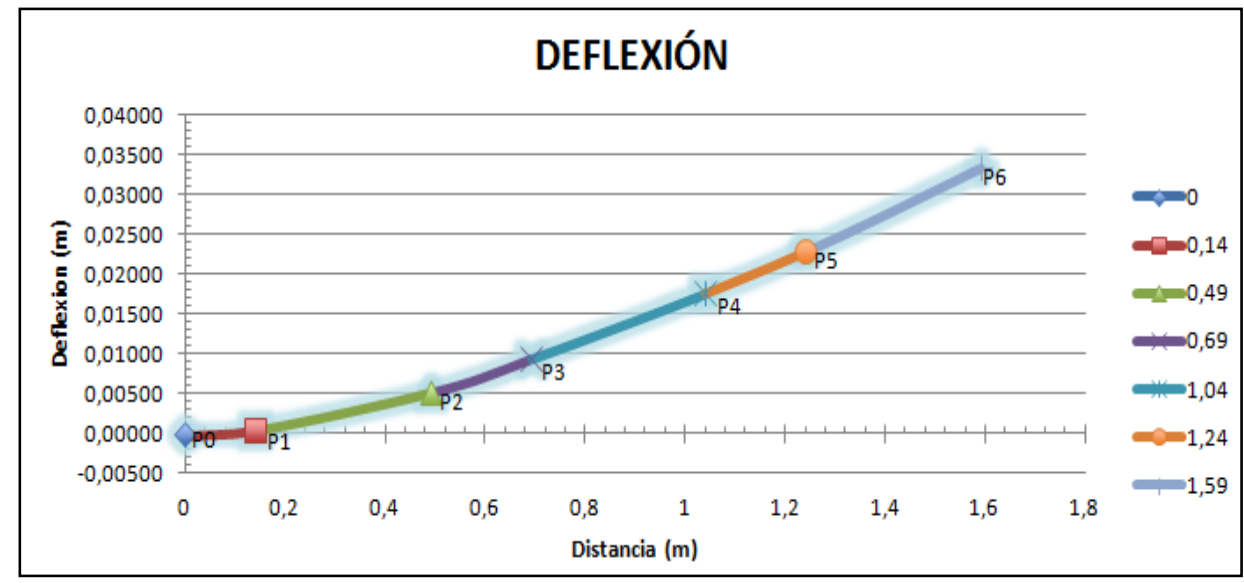

Fuente: Grupo de Investigación.

\section{c) Prueba II a 2 G de carga.}

En esta prueba se consideró el peso del avión sobre una de las alas multiplicado por un factor de carga de $2 \mathrm{G}$ que es $4410 \mathrm{~N}$ y la carga muerta de la distribución de fuerzas de 1445,7 N. Para poder determinar la fuerza que debe ejercer el cilindro hidráulico es necesario sumar la fuerza sobre la probeta ms la carga muerta de la distribución de fuerzas obteniendo 5855,7 N.

En la tabla 12 se puede observar los datos de la carga que debe soportar la probeta a $2 \mathrm{G}$ del peso del avión UAV.

Tabla 12: Fuerza y presión estimada para el ensayo

\begin{tabular}{cccccc}
\hline & $\begin{array}{c}\text { PRESION } \\
\text { CILINDRO }\end{array}$ & $\begin{array}{c}\text { FUERZA } \\
\text { CILINDRO }\end{array}$ & \multicolumn{2}{c}{$\begin{array}{c}\text { } \text { F FUERZA } \\
\text { SENSORES }\end{array}$} & $\begin{array}{c}\text { CARGA } \\
\text { MUERTA }\end{array}$ \\
\hline $\mathbf{n}$ & $(\mathrm{Pa})$ & $(\mathrm{N})$ & $(\mathrm{Lb})$ & $(\mathrm{N})$ & $(\mathrm{N})$ \\
$\mathbf{2 G}$ & $12,8 \times 10^{6}$ & $\mathbf{5 8 5 5 , 7}$ & 1160 & 5159,94 & 1445,7
\end{tabular}

Fuente: Grupo de Investigación. 
La tabla 13 se indica los valores de la carga distribuida obtenidos en las seis celdas de carga en la pantalla de Excel en función del tiempo de la prueba a 5855,7 N.

Tabla 13: Fuerza en las celdas de carga.

\begin{tabular}{|c|c|c|c|c|c|c|}
\hline \multicolumn{7}{|c|}{ TABLA GENERAL DE VALORES OBTENIDOS EN EL TIEMPO } \\
\hline & SENSOR1 & SENSOR2 & SENSOR3 & SENSOR4 & SENSOR5 & SENSOR6 \\
\hline to1 & 66 & 41 & 8 & 1 & -3 & -2 \\
\hline to2 & 162 & 127 & 83 & 54 & 32 & 17 \\
\hline to3 & 225 & 182 & 132 & 101 & 66 & 55 \\
\hline to4 & 230 & 218 & 167 & 130 & 90 & 80 \\
\hline tos & 230 & 241 & 193 & 151 & 105 & 97 \\
\hline to6 & 228 & 241 & 204 & 165 & 117 & 108 \\
\hline 107 & 228 & 241 & 218 & 173 & 124 & 117 \\
\hline to8 & 228 & 240 & 225 & 180 & 130 & 123 \\
\hline to9 & 228 & 241 & 230 & 186 & 135 & 125 \\
\hline $\mathrm{t} 10$ & 228 & 240 & 237 & 187 & 137 & 131 \\
\hline
\end{tabular}

Fuente: Grupo de Investigación.

En la tabla 14 se muestra los datos de las fuerzas obtenidas de las celdas de carga, las fuerzas cortantes y momento flector en los seis puntos distribuidos a lo largo de la probeta.

Tabla 14: Análisis de los resultados de la prueba.

\begin{tabular}{cccccc}
\hline PUNTOS & DISTANCIA & $\begin{array}{c}\text { FUERZA } \\
\text { SENSORES }\end{array}$ & $\begin{array}{c}\text { FUERZAS } \\
\text { CORTANTES }\end{array}$ & MOMENTOS & DEFLEXION \\
\hline & $\mathbf{( m )}$ & $\mathbf{( N )}$ & $\mathbf{( N )}$ & $\mathbf{( N m})$ & $\mathbf{( m )}$ \\
\hline P0 & 0 & - & $-4385,945$ & 2485,3688 & 0,0000 \\
P1 & 0,14 & 1014,19 & $-3693,300$ & 1920,5157 & 0,0013 \\
P2 & 0,49 & 1067,57 & $-2221,959$ & 896,1902 & 0,0125 \\
P3 & 0,69 & 1054,23 & $-1548,132$ & 521,2045 & 0,0221 \\
\hline P4 & 1,04 & 831,82 & $-661,079$ & 145,4373 & 0,0418 \\
P5 & 1,24 & 609,41 & $-321,130$ & 49,2399 & 0,0542 \\
P6 & 1,59 & 582,72 & $-18,363$ & 0,6733 & 0,0795 \\
\hline
\end{tabular}

Fuente: Grupo de Investigación. 


\section{c.1) Distribución de fuerzas.}

En la figura 11 se observa la fuerza con respecto a la distancia obteniendo una gráfica que cumple con la distribución de fuerzas que sea mayor en el empotramiento y vaya disminuyendo hacia el extremo libre de la probeta.

Figura 11: Distribución de fuerzas.

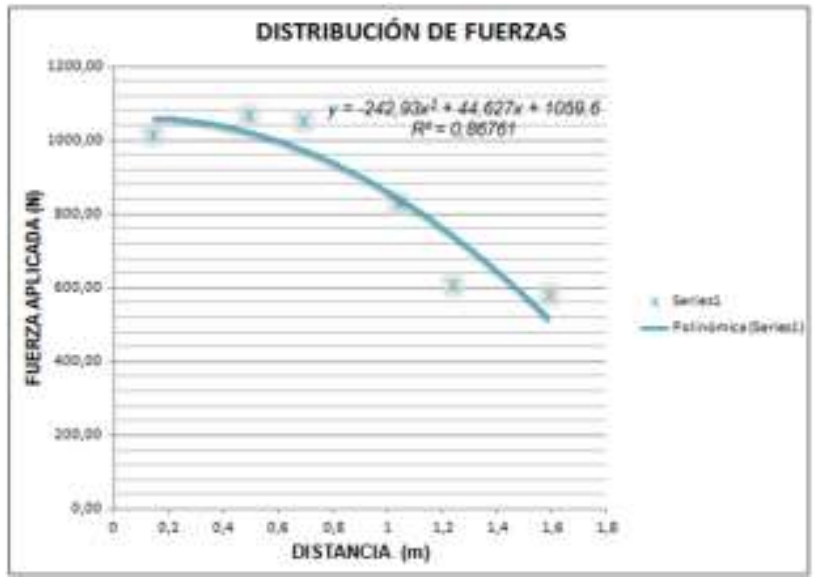

Fuente: Grupo de Investigación.

\section{c.2) Fuerzas cortantes.}

En la figura 12 determinamos que el punto más crítico es en el empotramiento P0 con esfuerzo cortante de $-4385,945 \mathrm{~N}$ y el punto $\mathrm{P} 1$ con $-3693,300 \mathrm{~N}$, debido al valor de su carga que se aplica en esa distancia.

Figura 12: Diagrama de fuerzas cortantes.

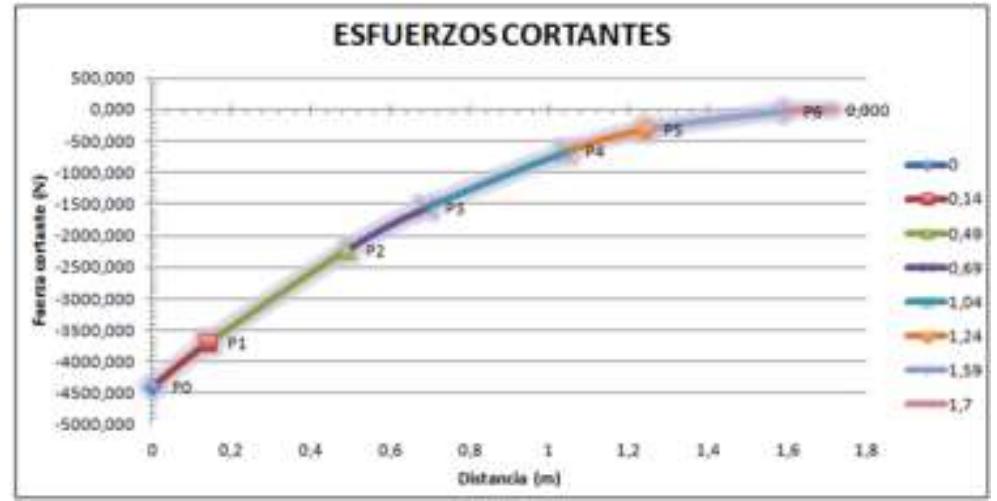

Fuente: Grupo de Investigación. 


\section{c.3) Momento flector.}

En la figura 13 determinamos que el punto más crítico es en el empotramiento $\mathrm{P} 0$ con un momento flector de 2485,3688 Nm y en el punto P1 de 1920,5157 Nm, debido a la carga que se aplica en esas distancias.

Figura 13: Diagrama de momento flector.

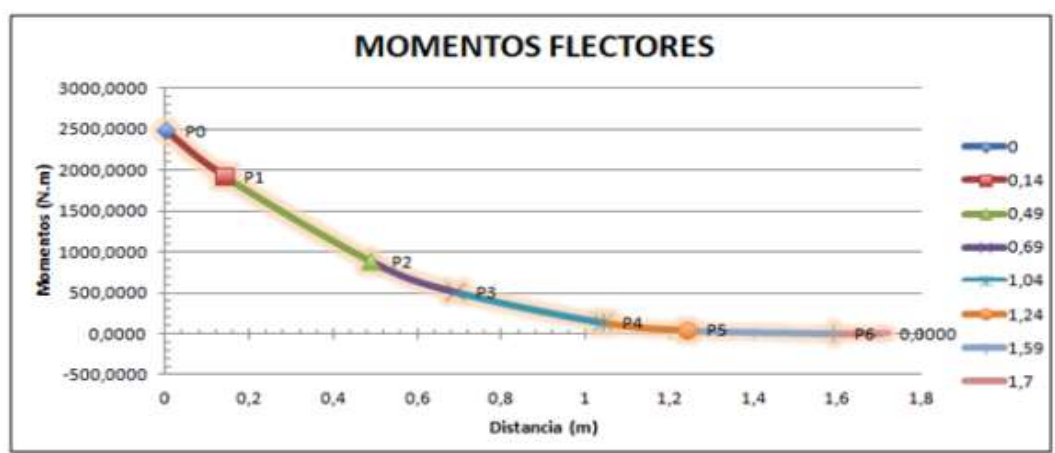

Fuente: Grupo de Investigación.

\section{c.4) Deflexión.}

En la figura 14 se observa que la deflexión máxima sobre la probeta de madera a una carga de $3683,18 \mathrm{~N}$ se produce en el punto seis obteniendo un valor de 0,0795 m.

Figura 14: Curva de la deflexión.

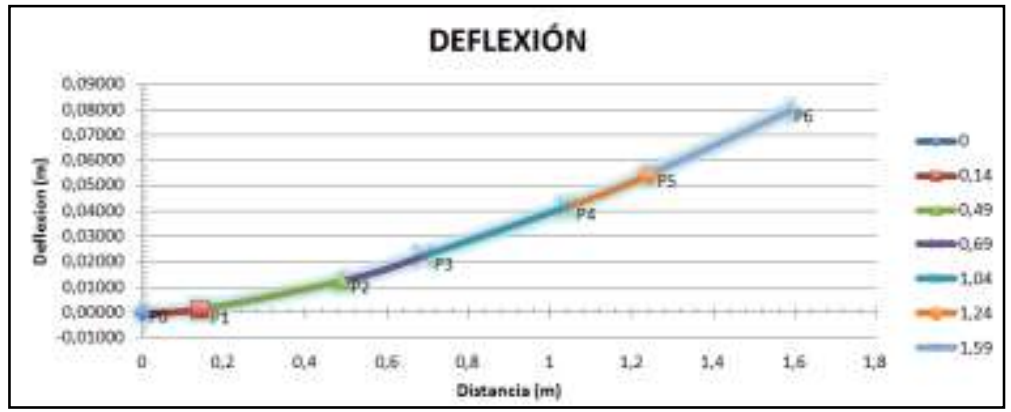

Fuente: Grupo de Investigación. 


\section{Conclusiones.}

- Las estructura del banco de pruebas para ensayos de resistencia estructural de alas y alerones soporta una carga máxima de $25151 \mathrm{~N}$, y se puede determinar apara una longitud de $1700 \mathrm{~mm}$ que garantiza el funcionamiento.

- Los modelos matemáticos para el dimensionamiento de los elementos constitutivos del banco de prueba se comparó con el análisis de elementos finitos con un factor de seguridad mínimo de 2 para estructuras bajo cargas estáticas, lo que da una idea clara de que el banco de pruebas podría trabajar con un rendimiento óptimo a la máxima carga.

- Los perfiles, tubo cuadrado y plancha de acero ASTM A36 utilizado en la construcción de la estructura del banco de pruebas ofrece las mejores características mecánicas, que permite un amplio margen de diseño de la estructura.

- El banco de pruebas para ensayos de resistencia estructural de alas permite obtener los valores de carga en seis puntos sobre el ala para determinar los valores de esfuerzo cortante, momento flector y deflexión, gracias a las seis celdas de carga colocadas sobre la probeta de prueba.

- El sistema hidráulico utilizado tiene una presión hidráulica de 13,79 MPa que genera una fuerza a tracción máxima de $25151 \mathrm{~N}$ necesaria para realizar las pruebas.

- El diseño de un módulo de control electrónico para el funcionamiento del banco de pruebas, se realizó en función de las necesidades de accionar la electroválvula que direcciona el aceite al cilindro hidráulico y el acondicionamiento de las celdas de carga.

\section{Referencias bibliográficas.}

- AISC. (2005). Manual of Steel Construction. 8th Edition. Chicago

- Coughlin \& Driscoll, R. F. (1999), Amplificadores Operacionales y Circuitos Integrados Lineales. Mexico D.F.: Prentice Hall

- Creus, N. E. (2011). Hidraulica. Mexico D.F.: Alfaomega

- Megson, \& Gordon, T. H. (2010). An Introduction to Aircraft Structural Analysis. London: ELSEVIER B.H.

- Saavedra, A. R. (1990). Manual del Piloto Privado (5 Edicion). Madrid-España: PILOT’S, S.A.Suministros Aeronáuticos

- Castaño, F. (En línea).Materiales Utilizados en la Industria Aeronautica. (Citado 1210-2014) de: http://es.scribd.com/doc/140981268/Materiales-Utilizados-en-laIndustria-Aeronautica-Fernando-Castano-Membrives

- INTERDEFENSA. UNASUR I. (Citado 15-12-2014) de: http://interdefensa.argentinaforo.net/t9239-el-entrenador-unasur-i-depura-sus-lineas.

- THALES. Sistemas y oluciones para vehículos aéreos no tripulados. (Citado 20-102014) de: 
https://www.thalesgroup.com/sites/default/files/asset/document/sistemas_y_solucio nes_para_vehiculos_aereos_no_tripulados.pdf 


\section{Para citar el artículo indexado.}

Torres G., Cruz M., Mena S., Torres G., Herrera T. \& Zambrano D. (2018). Diseño y construcción de un banco de pruebas para ensayos de resistencia estructural de alas y alerones. Revista Electrónica Ciencia Digital 2(1), 88-104. Recuperado desde: http://www.cienciadigital.org/revistascienciadigital/index.php/CienciaDigital/article/view/7 $/ 7$

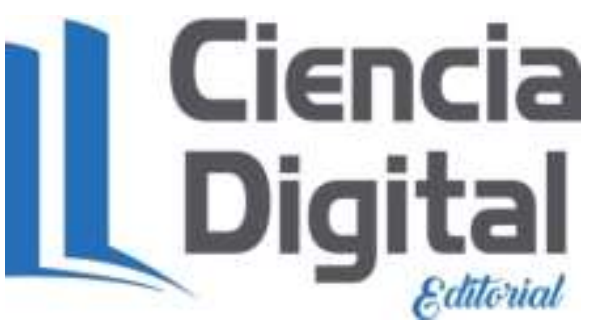

El artículo que se publica es de exclusiva responsabilidad de los autores y no necesariamente reflejan el pensamiento de la Revista Ciencia Digital.

El articulo queda en propiedad de la revista y, por tanto, su publicación parcial y/o total en otro medio tiene que ser autorizado por el director de la Revista Ciencia Digital.
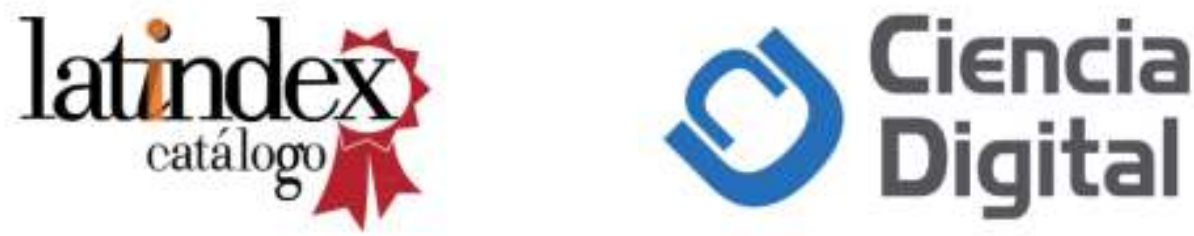